\title{
Imaging capabilities of hypertelescopes with a pair of micro-lens arrays
}

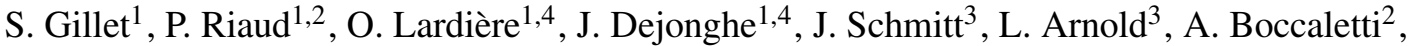 \\ D. Horville ${ }^{5}$, and A. Labeyrie ${ }^{1,4}$
}

1 LISE-Observatoire de Haute-Provence, 04870 St Michel l'Observatoire, France e-mail: sgohp@obs-hp.fr; lardiere@obs-hp.fr; dejonghe@obs-hp.fr; riaud@obs-hp.fr

2 LESIA, Observatoire de Paris-Meudon, 5 Pl. J. Janssen, 92195 Meudon, France e-mail: pierre.riaud@obspm.fr; anthony.boccaletti@obspm.fr

3 Observatoire de Haute-Provence, 04870 St Michel l'Observatoire, France e-mail: schmitt@obs-hp.fr; arnold@obs-hp.fr

${ }^{4}$ Collège de France, 11 Pl. M. Berthelot, 75321 Paris, France e-mail: labeyrie@obs-hp.fr

5 GEPI - Observatoire de Paris, 61 avenue de l'Observatoire, 75014 Paris, France e-mail: david.horville@obspm.fr

Received 29 May 2002 / Accepted 15 November 2002

\begin{abstract}
We verify the imaging performance of hypertelescopes on the sky, using a new scheme for pupil densification. To avoid seeing limitations, we used a miniature version with a $10 \mathrm{~cm}$ aperture containing 78 sub-apertures of $1 \mathrm{~mm}$ size, arrayed periodically as a square grid. The pupil densification is achieved with a pair of micro-lens arrays, where each pair of facing lenses behaves like a tiny demagnifying telescope. We have tested the direct snapshot performance with laboratory-simulated multiple stars and observed the binary star Castor $(\alpha \mathrm{Gem})$. We measured a separation of $3.8^{\prime \prime}$ and a magnitude difference of 0.85 which is in agreement with current orbital data. This verified the theoretical expectations for hypertelescopes in terms of field of view and fluxes and qualified the new optical implementation for future arrays at the scale of meters and beyond.
\end{abstract}

Key words. instrumentation: interferometers - techniques: high angular resolution - stars: binaries: visual methods: observational

\section{Introduction}

Very large telescopes are needed for imaging stellar disks or exo-planets but it will be difficult to expand the size beyond that of current "Extremely Large Telescope" projects such as the $30 \mathrm{~m}$ CELT and the $100 \mathrm{~m}$ OWL (Gilmozzi et al. 1998), for technological and cost reasons. Interferometry can provide various trade-offs between angular resolution and collecting area, but snapshot imaging is often desirable to observe short-lived phenomena. In 1996, Labeyrie (1996) described the possibility of making snapshot images with multi-aperture interferometers, using a densified pupil. This optical architecture allows direct high-resolution imaging with high contrast. A miniature instrument, using a diffractive approach to pupil densification, has been successfully tested by Pedretti et al. (2000) on bright binary stars. Now, we present results similarly obtained with an improved optical scheme for achieving the pupil densification. In Sects. 2 and 3 we describe the principle of hypertelescopes and the experimental setup. Section 4 presents the results obtained on the sky. And finally, the optical schemes are further discussed in Sect. 5.

Send offprint requests to: S. Gillet, e-mail: sgohp@obs-hp. fr

\section{Principle}

The principle of densified-pupil multi-aperture imaging interferometers, called hypertelescopes, was previously described (Labeyrie 1996; Gillet et al. 2001; Riaud et al. 2002). Let us consider light beams coming from $N$ small mirrors arrayed periodically in two dimensions with period $s$. Beams from these mirrors are initially combined according to a Fizeau interferometer geometry. At the combined focus, the image has a central peak surrounded by many secondary dispersed peaks. Densifying the entrance pupil, shrinks and intensifies the combined image, thus attenuating or suppressing the secondary peaks. The densified pupil increases the limiting magnitude but limits the field of view to a non-aliased field of the interferometer.

The white central peak, in the densified-pupil image, appears only for stars within a small region of the sky. Following Gillet et al. (2001), we call ZOF (Zero Order Field) this narrow usable field and HOF (High Order Field) the peripheral sky field of size $\lambda / d$ where $d$ is the size of one sub-aperture $(1 \mathrm{~mm})$ :

$Z O F(\mathrm{sky})=\frac{\lambda}{s}=1.22 \frac{\lambda}{d \cdot \gamma_{\mathrm{D}}}$ 


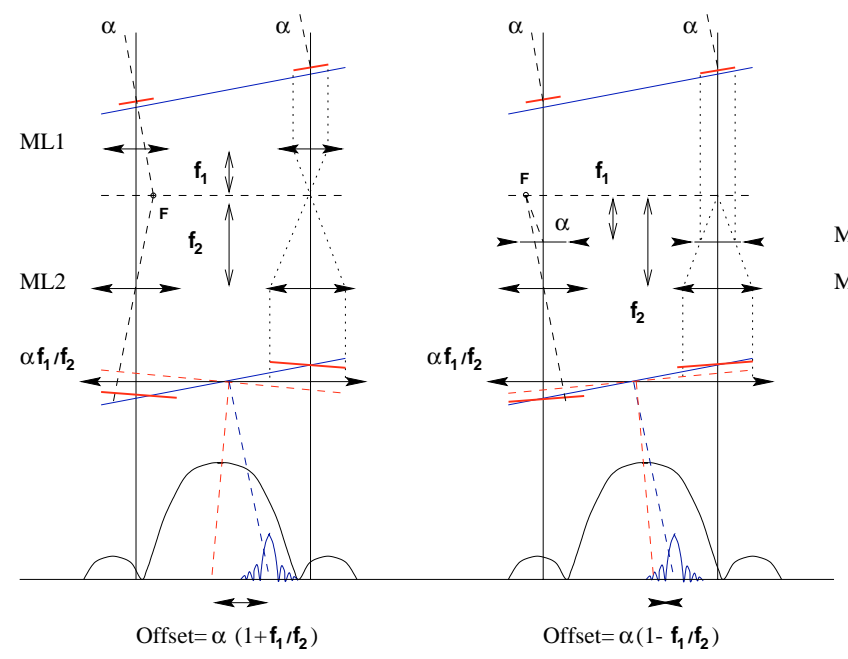

Fig. 1. Hypertelescope principle. Positive (left) and negative (right) pupil densification, using respectively a diverging and a converging lens array, followed in each case by a longer-focus array of converging lenses. A tilted incoming wavefront from an off-axis star is densified with two confocal lens arrays (ML1 and ML2), thus producing a densified wavefront which focuses to a single narrow interference peak within the broader diffraction pattern from each sub-pupil. It becomes stair-shaped since the slope of each wavefront segment is reduced by the pair of micro-lenses. The stair's average shape remains flat and parallel to the incoming wavefront since all pairs of micro-lenses introduce identical propagation delays. But each wavefront segment, arriving with a slope angle $\alpha$, emerges with an angle $\alpha * f 1 / f 2$ where $f 1$ and $f 2$ are the micro-lens's focal lengths of ML1 and ML2. The resulting wavefront is finally a stair-shaped wavefront with the same average slope as at the entrance of the densifier.

where $\gamma_{D}$ the densification ratio. This densification ratio is defined by:

$\gamma_{\mathrm{D}} \equiv \frac{\left(\frac{B}{d}\right)_{i}}{\left(\frac{B}{d}\right)_{\mathrm{o}}}=\frac{d_{\mathrm{o}}}{d_{i}}$ if $B_{i}=B_{\mathrm{o}}$

where $d_{i}$ and $d_{\mathrm{o}}$ are the entrance mirror and exit sub-pupil diameters respectively, and $B_{i}$ and $B_{\mathrm{o}}$ the entrance and exit baselines in the pupil densifier. In the configuration considered here (Fizeau interferometer), both baselines are equal. But in case of a Michelson interferometer, the entrance baseline $B_{i}$ is different from the output baseline $B_{0}$.

The number of resolution elements in the ZOF is given by:

$N_{\text {resels }}=\left(B /\left(d \cdot \gamma_{\mathrm{D}}\right)\right)^{2}$

$B$ is the baseline of the interferometer (here $10 \mathrm{~cm}$ ).

\section{Experimental arrangement}

Following the initial sky testing achieved by Pedretti et al. (2000), using a diffractive mode of pupil densification, we have tried to improve the densification technique. Among a wide range of possibilities, we have chosen to use a pair of micro-lens arrays. The hypertelescope's scheme is represented in Fig. 2. Instead of a true array of mirror elements, we have used a $40 \mathrm{~cm}$ Newtonian telescope at Observatoire

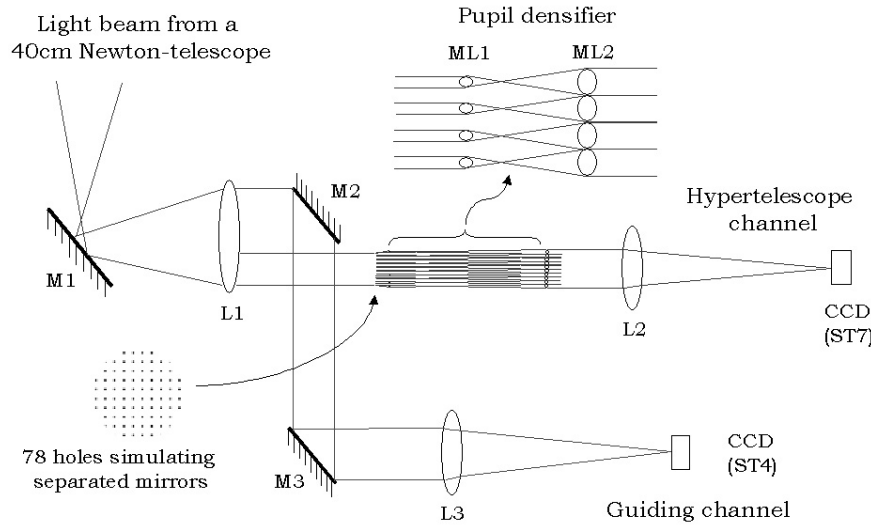

Fig. 2. Hypertelescope experimental setup. The incoming light beam from a Newtonian telescope is collimated by lens L1. A Fizeau mask installed for convenience in the pupil plane following L1, rather than at the primary mirror, has $N=78$ holes of $100 \mu \mathrm{m}$ size each. It defines in the entrance aperture a virtual "diluted giant mirror" of $10 \mathrm{~cm}$ size with $s=1 \mathrm{~mm}$ sub-apertures. The densification is achieved with two micro-lens arrays $\left(\mathrm{ML}_{1}\right.$ and $\left.\mathrm{ML}_{2}\right)$.

de Haute Provence as collecting optics. In order to have nearly diffraction-limited image quality without adaptive optics, the hypertelescope exploits only $10 \mathrm{~cm}$ of the aperture. The rest of the aperture served for auto-guiding with a SBIG ST4 CCD camera.

The lens L1 produces a pupil image, 10 times smaller than the entrance aperture, which is masked by a grid of 78 holes of $0.1 \mathrm{~mm}$ size, centered $1 \mathrm{~mm}$ apart. The virtual grid thus defined in the entrance aperture has $1 \mathrm{~mm}$ holes spaced $10 \mathrm{~mm}$ apart. Two arrays of convergent and confocal micro-lenses (ML1 and ML2), having a short and a long focal length respectively (20 mm and $120 \mathrm{~mm}$ ), achieve the pupil densification. The front focal plane of the first array is located close to the grid, so as to provide a pupil plane close to the second array. Collimated beams from each sub-pupil become recollimated and widened when transmitted through the facing pair of micro-lenses. The densification factor, ratio of ML2 and ML1's focal lengths, amounts to 6 , providing $80 \%$ filling (diameter) in the exit pupil. The micro-lens arrays utilized were fabricated by one of us $(\mathrm{DH})$ at Observatoire de Paris (Bensammar et al. 2000), with enough lens-to-lens uniformity of thickness to keep piston errors within Rayleigh's tolerance, as required for a highly constructive interference, providing a high Stehl ratio, in the star's "high-resolution" image. The rather faint star images thus formed with less than $1 \mathrm{~cm}^{2}$ of total collecting area were recorded on a CCD camera with $9 \mu \mathrm{m}$ pixels (0.62 pixel/" image sampling). With its aperture size of $10 \mathrm{~cm}$ and equivalent mirrors of $1 \mathrm{~mm}$ diameter, the ZOF extent is $1.22(\lambda / d) / \gamma_{\mathrm{D}}=28^{\prime \prime}$ at $\lambda=675 \mathrm{~nm}$ and the angular resolution is $1.22 \lambda / B=1.6^{\prime \prime}$.

\section{Laboratory and sky results}

We first tested the imaging capabilities of the hypertelescope with laboratory-simulated single or multiple point sources. Figure 4 shows the laboratory images obtained. The classical limitation of interferometers regarding field crowding also 


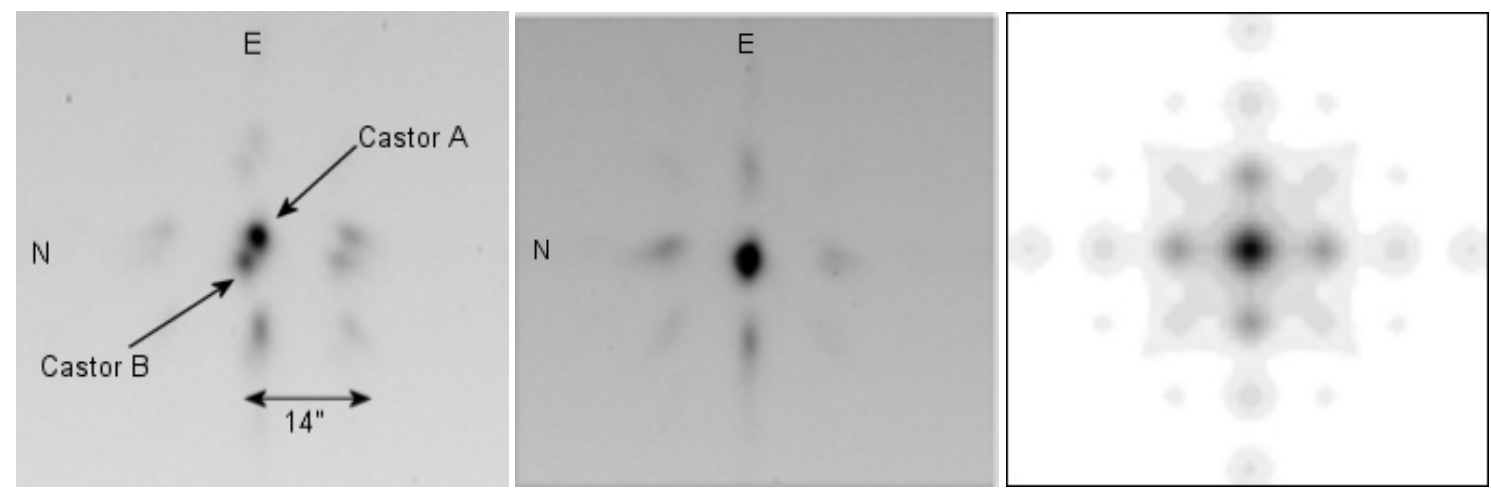

Fig. 3. a) Image of Castor, showing the resolved binary A-B, spaced 3.8 arcsec. The half ZOF is about $14 \pm 0.6$ arcsec wide. b) Image of Pollux, obtained with a $10 \mathrm{~min}$ exposure. It matches the theoretical pattern, with the residual side-peaks due to incomplete pupil densification. With respect to the laboratory images and the numerical simulation, the peaks are however somewhat widened by seeing and exceed the theoretical arc-second resolution limit of the $10 \mathrm{~cm}$ array. c) Numerical simulation of a point source's monochromatic image with the 78-aperture hypertelescope.

arises in hypertelescopes. With a periodic array of $N$ elements, field crowding occurs when the ZOF contains more than $4 N$ stars. The image is aliased by dispersed higher-order peaks from those stars located in the HOF but outside of the ZOF, thus affecting the information retrieval. No such degradation occurs here with the numerous apertures and the few stars.

In a second step, we verified the hypertelescope's imaging properties on bright stars. Figure $3 b$ shows an image of Pollux ( $\beta \mathrm{Gem}, m_{\mathrm{v}}=1.14$ ) exposed $10 \mathrm{~min}$. The four residual peaks of order 1 surrounding the central zero-order peak result from the incomplete densification utilized. Their measured intensity relative to the central peak is $1 / 16$, close to the theoretical value $1 / 20$. In October 2001, we have obtained images of binary star Castor A-B $\left(\alpha\right.$ Gem, $\left.m_{\mathrm{v}}=1.98\right)$, for which the magnitude difference is $\Delta m=0.9$. Exposures were taken with and without Wratten filters W25 $(R)$, W58 $(G)$ and W80A $(B)$ during 10 to $30 \mathrm{~min}$. In Fig. $3 \mathrm{c}$ the companion $\alpha$ Gem B appears clearly, in spite of seeing affecting the image's sharpness. We measured for JD $=2452193.67$ a separation $\rho=3.8 \pm 0.3 \operatorname{arcsec}$ and a position angle $\alpha=68.2 \pm 5^{\circ}$, close to the expected values (Heintz 1988), $\rho=4.0$ arcsec and $\alpha=63.81^{\circ}$. The theoretical number of resolution elements inside the ZOF is $312(4 N)$. We measured in Figs. 3b and 3c, 307 \pm 26 resolutions elements (see Eq. (3)), due to uncertainly of ZOF diameter $(28 \pm 1.2 \operatorname{arcsec})$ mesurement.

The intensity profile of Castor A contaminates the flux of Castor B by about $20 \%$. After appropriate subtraction, the magnitude difference between Castor $\mathrm{A}$ and Castor $\mathrm{B}$ is $\Delta m=$ $0.8 \pm 0.15$. The significant magnitude inaccuracy is due to a nearly 2.7 "FWHM seeing.

\section{Discussion}

Several optical schemes are possible for the pupil densifier. With respect to the diffractive mode of pupil densification tested by Pedretti et al. (2000), using a single micro-lens array, the present optical scheme adopted here, with two micro-lens arrays, is more flexible and gives a better control of sub-pupil
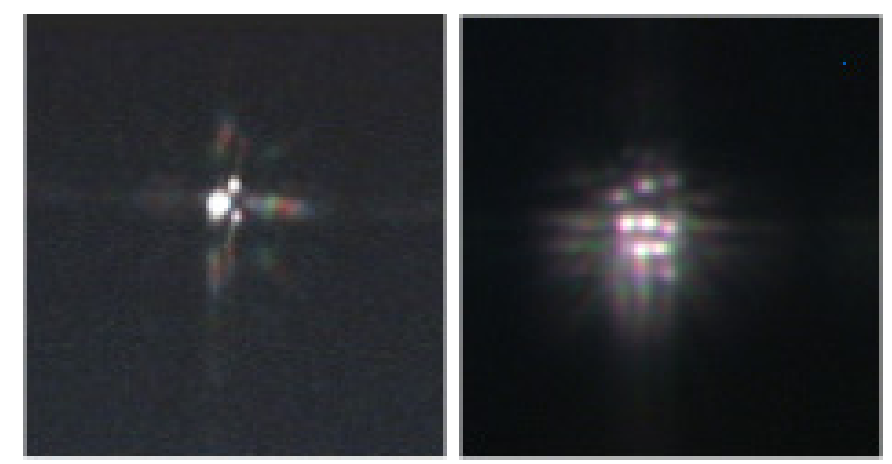

Fig. 4. Laboratory simulated triple (left) and quintuple (right) stars observed with the hypertelescope. The image is a color composite of three images taken through $R, G$ and $B$ filters.

filling. The densified pupil seen from the camera, although only $80 \%$ densified in this experiment, can in principle be more completely filled to obtain a rather uniform wavefront with very narrow gaps, of interest for coronagraphy.

The alignment however is more difficult than with a single micro-lens array. The pair of micro-lens arrays indeed requires a careful rotational alignment of both arrays relative to the aperture grid within $1^{\circ}$. Misalignment causes a novel form of aberration: the main rays from sub-pupils define a series of co-axial single-sheet hyperboloids, instead of co-axial cylinders. This is evidenced as a rotation of the image when the camera is moved in and out of focus. Both micro-lens arrays had rotating mounts for this adjustment. The final image must appear (in white light) as a central white peak, surrounded by symmetrical secondary dispersed peaks if densification is incomplete.

We used converging micro-lenses for the first array although diverging ones in principle provide a wider $\mathrm{ZOF}$ since the image's diffractive envelope then moves in the same direction as the interference peak. Indeed, with diverging lenses, a point source located at field angle $\alpha$, gives a central interference peak which is off-set as $\alpha\left(1-1 / \gamma_{\mathrm{D}}\right)$ (Fig. 1). With converging lenses instead, the off-set goes as $\alpha\left(1+1 / \gamma_{\mathrm{D}}\right)$. 


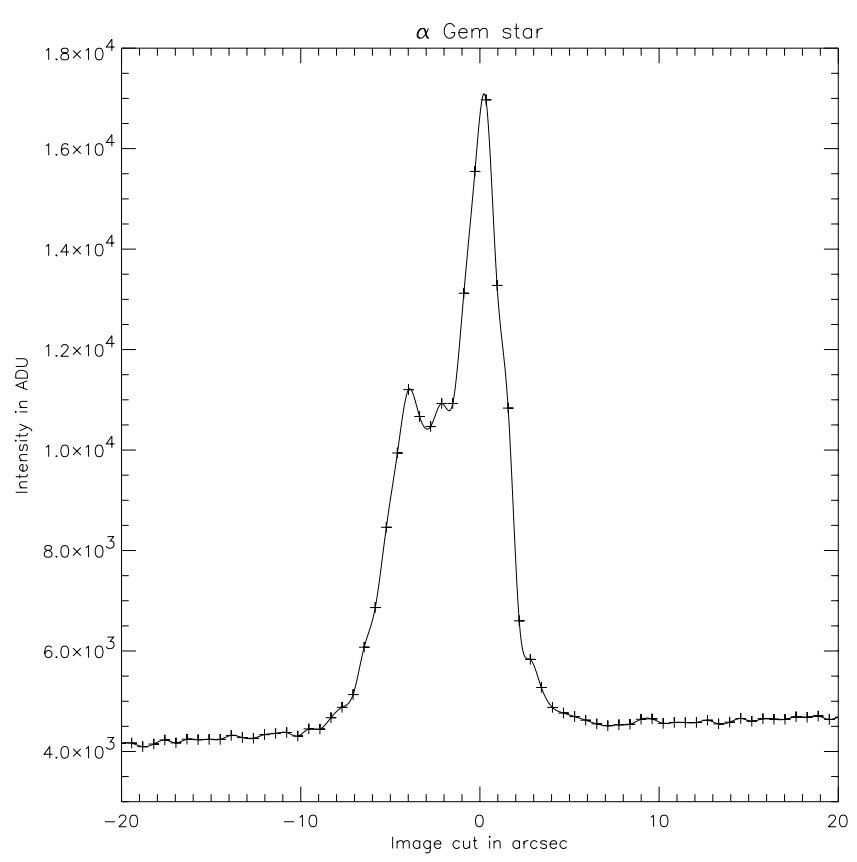

Fig. 5. Profile of the image of Castor A-B, showing the resolved companion. The Strehl ratio is degraded by seeing.

The difference however becomes vanishingly small with increasing values of the densification factor. With the densification value adopted here, the converging lenses of the first array only cause a minor reduction of field size. Two points are indeed critical for direct imaging. First of all, it is not possible to image more than $4 \mathrm{~N}$ sources (for our configuation) corresponding to the number of resolution elements contained in the ZOF. Second of all, any object outside the ZOF (28" in diameter), but within the HOF ( $2^{\prime} 42^{\prime \prime}$ in diameter) appears in the ZOF owing to its dispersed higher-order peaks. This effect, called confusion noise, reduces the signal to noise ratio. If the sources density is weak, it becomes possible to reconstruct an image, using multi-spectra exposures (three wavelengths or more) in order to determine the position of objects outside the ZOF. This would increase the field of view of a densified pupil interferometer. The confusion noise induces errors in the determination of the source positions. With 312 resels, we can observe a density of 54 sources per squared arcmin.

\section{Conclusion and future work}

The sky testing with the new densification scheme confirmed the high-resolution imaging capabilities of hypertelescopes and their applicability to observing methods highly sensitive to the exit pupil shape, such as phase-mask coronagraphy. The image quality has been noticeably improved. The filling and uniformity of the exit pupil is indeed easily controllable, and the densifier system can be a compact package installed at the Fizeau focus of a large multi-mirror array. Its use is straightforward if adaptive optics provides the required piston phasing and also phasing within each sub-aperture if their size exceeds Fried's parameter. Un-phased images can be exploited by speckle interferometry. Direct imaging occurs within the unaliased narrow field of view (ZOF) and the images are usable for spectroscopic, spectro-imaging and also coronagraphic uses with a focal mask such as Roddier's phase dot or Rouan's Four Quadrant Phase Mask (Riaud et al. 2001). A hypertelescope coupled to a coronagraph has been proposed for NASA's Terrestrial Planet Finder project (Riaud et al. 2002). The concept is being further explored in our group. At given collecting area, more apertures of smaller size indeed enlarge the direct field (ZOF) and more sources are tolerable within the HOF (the size of which increases). Thus, current designs of Extremely Large Telescopes can be "exploded" for higher resolution with the same limiting magnitude, while retaining their deep-field imaging capability: this announces ground and space interferometric instruments with powerful observing characteristics.

\section{References}

Bensammar, S., Gex, F., Horville, D., et al. 2000, Next Generation Space Telescope Science and Technology, ASP Conf. Ser., 207, 412

Gillet, S., et al. 2001, CR. Acad. Sci. Paris, t. 2, Serie IV, 27

Gilmozzi, R., et al. 1998, SPIE, 3352, 778

Heintz, W. D. 1988, PASP, 100, 834

Labeyrie, A. 1996, A\&AS, 118, 517

Pedretti, E., Labeyrie, A., Arnold, L., et al. 2000, A\&AS, 147, 285

Riaud, P., Boccaletti, A., Rouan, D., Lemarquis, F., \& Labeyrie, A. 2001, PASP, 113, 1145

Riaud, P., Gillet, S., Labeyrie, A., et al. 2001, Proc. From Optical to Millimetric Interferometry, Liège Int. Astroph. Coll.

Riaud, P., Boccaletti, A., Gillet, S., et al. 2002, A\&A, 396, 345 